\title{
Estimação da área foliar de canola por dimensões foliares
}

\author{
Alberto Cargnelutti Filho ( $\left.{ }^{*}\right)$; Marcos Toebe (2); Bruna Mendonça Alves ( $\left.{ }^{1}\right)$; Cláudia Burin (1); \\ Jéssica Andiara Kleinpaul (') \\ (1) Universidade Federal de Santa Maria (UFSM), Departamento de Fitotecnia, Avenida Roraima, 1000, 97105-900 Santa Maria (RS), \\ Brasil. \\ (2) Universidade Federal do Pampa (UNIPAMPA), Rua Luiz Joaquim de Sá Britto, s/n, 97650-000 Itaqui (RS), Brasil. \\ (*) Autor correspondente: alberto.cargnelutti.filho@gmail.com
}

Recebido: 4/nov./2014; Aceito: 5/jan./2015

\begin{abstract}
Resumo
O objetivo deste trabalho foi modelar e identificar os melhores modelos para a estimação da área foliar determinada por fotos digitais, de três híbridos de canola, em função do comprimento, ou da largura e/ou do produto comprimento vezes largura do limbo foliar. Foram conduzidos três ensaios de uniformidade com canola (Brassica napus L.), e em cada ensaio avaliou-se um dos seguintes híbridos: Hyola 61, Hyola 76 e Hyola 433. Em cada híbrido, foram coletadas 125 folhas aos 77, 84, 91 e 97 dias após a semeadura, totalizando 1.500 folhas. Nessas 1.500 folhas, foram mensurados o comprimento (C) e a largura (L), e calculado o produto do comprimento vezes a largura $(C \times L)$ do limbo foliar. Determinou-se a área foliar de cada folha, por meio do método de fotos digitais (Y). Posteriormente, para cada híbrido, foram separadas, aleatoriamente, $80 \%$ das folhas ( 100 folhas por coleta $\times 4$ coletas por híbrido $=400$ folhas por híbrido) para a geração de modelos do tipo quadrático, potência e linear, de Y em função do C, da L, e/ou do C×L. Os 20\% das folhas restantes (100 folhas por híbrido) foram usadas, separadamente, para a validação dos modelos. Em canola, os modelos do tipo potência, para os híbridos Hyola $61\left(\hat{Y}=1,3000 x^{1,9336}, R^{2}=0,9531\right)$, Hyola $76\left(\hat{Y}=1,0579 x^{2,0383}, R^{2}=0,9733\right)$ e Hyola $433\left(\hat{Y}=1,4154 x^{1,9096}, R^{2}=0,9613\right)$, são adequados para a estimação da área foliar determinada por fotos digitais (Y) em função da largura do limbo foliar ( $\mathrm{x}$ ).
\end{abstract}

Palavras-chave: Brassica napus L., fotos digitais, modelagem, método não destrutivo.

\section{Leaf area estimation of canola by leaf dimensions}

\begin{abstract}
The objective of this work was to model and identify the best models to estimate the leaf area determined by digital photos, of three canola hybrid, with the length or width and / or the product length width of the leaf. Three uniformity trials were carried with the culture of canola (Brassica napus L.). In each trial was valued one of the following hybrids: Hyola 61, Hyola 76, Hyola 433. In each hybrid were collected 125 leaves at 77, 84, 91, 97 days after sowing, totaling 1,500 leaves. In these 1,500 leaves were measured length $(C)$ and width $(L)$ and calculated the length width $(C \times L)$ of the leaf. Was determined the leaf area of each leaf, by the method of digital photos (Y). After, for each hybrid were separated, randomly, $80 \%$ of the leaves (100 leaves by collects $\times 4$ collects by hybrid $=400$ leaves per hybrid) to build models of quadratic, potency and linear type for $Y$ function of the C, $L$ and/or $C \times L$. The remaining $20 \%$ of the leaves (100 leaves by hybrid), separately, were used to validate the models. In canola, the potency model for hybrid Hyola $61\left(\hat{Y}=1.3000 x^{1.9336}, R^{2}=0.9531\right)$, Hyola $76\left(\hat{Y}=1.0579 x^{2.0383}, R^{2}=0.9733\right)$ and Hyola $433\left(\hat{Y}=1.4154 x^{1.9096}, R^{2}=0.9613\right)$, are adequate for estimation of leaf area determined by digital photos $(Y)$ as function of the width of the leaf $(\mathrm{x})$.
\end{abstract}

Key words: Brassica napus L., digital photos, modeling, non destructive method.

\section{INTRODUÇÃO}

A canola (Brassica napus L.), pertencente à família Brassicaceae, é uma oleaginosa que foi desenvolvida por meio do melhoramento genético da colza. No Brasil, é utilizada para a produçáo de grãos, em cultivo de inverno no sistema de rotação de culturas, contribuindo para a reduçáo de problemas fitossanitários de leguminosas, como a soja e o feijão, e de gramíneas, como o milho e o trigo. Também é utilizada na produção de óleo comestível e biodiesel, e seus resíduos são destinados à elaboração de raçôes animais (Tomm et al., 2009). 
A área foliar é um importante indicador empregado na análise de crescimento vegetal. Nesse sentido, a quantificação da área foliar por meio de métodos diretos requer equipamentos caros e/ou técnicas destrutivas, que impedem a mensuração do crescimento das folhas ao longo de determinado período de tempo. Diante disso, torna-se importante a estimação da área foliar por meio de métodos indiretos e não destrutivos, como pela utilização de equações matemáticas previamente desenvolvidas para cada espécie. As equaçóes matemáticas são, em geral, consideradas precisas, de fácil uso e possibilitam a rápida execução das medidas a campo, permitindo avaliaçóes sucessivas durante o crescimento de determinada espécie (Zucoloto et al., 2008).

Para a geração de equações matemáticas, utilizam-se modelos que descrevem a relação existente entre as dimensôes lineares das folhas (comprimento, largura ou produto comprimento vezes largura) e a área total destas. Modelos de estimação da área foliar em função das dimensôes lineares das folhas já foram desenvolvidos em diversas culturas, como em meloeiro (Nascimento et al., 2002), couve-folha (Marcolini et al., 2005), pinhão-manso (Severino et al., 2007), bananeira (Zucoloto et al., 2008), cafeeiro (Flumignan et al., 2008), feijāo-caupi (Lima et al., 2008), girassol (Maldaner et al., 2009; Aquino et al., 2011), batata (Busato et al., 2010), crambe (Toebe et al., 2010), videira (Borghezan et al., 2010), feijão-de-porco (Toebe et al., 2012), mucuna-cinza (Cargnelutti et al., 2012a) e nabo-forrageiro (Cargnelutti et al., 2012b).

Em estudos de modelagem da área foliar em função das dimensôes lineares das folhas têm sido utilizados aparelhos integradores de área foliar na quantificação da área foliar real. No entanto, recentemente, o processamento de imagens digitais (fotos digitais) vem sendo empregado para esse fim. Nesse sentido, Flumignan et al. (2008) verificaram que o método de imagens digitais pode substituir o método integrador de área foliar sem perda de qualidade, sendo considerado preciso, não destrutivo, de baixo custo e portátil. Ainda, Godoy et al. (2007) verificaram que o método de determinação da área foliar por imagem de câmera digital é mais preciso, apresenta maior coeficiente de determinação, menor custo e maior funcionalidade a campo e em laboratório, em relação aos integradores de área foliar. $\mathrm{O}$ uso de imagens digitais para a quantificação da área foliar real em estudos de modelagem da área foliar em função das dimensôes lineares das folhas também foi empregado nos estudos conduzidos por Toebe et al. (2010, 2012) e Cargnelutti et al. (2012a, b).

Segundo Pinto et al. (2008), o formato da folha é uma característica morfológica específica de cada espécie vegetal, e a relação entre as dimensões lineares e a área foliar depende da quantidade de recortes na borda do limbo foliar, entre outros fatores. Segundo os autores, é necessária a geração de modelos específicos de estimação da área foliar para cada espécie vegetal. Além disso, dentro de uma espécie vegetal, diferentes modelos podem ser necessários de acordo com a variabilidade genética existente entre as cultivares. Nesse sentido, Borghezan et al. (2010) constataram que, em videira, cada variedade apresenta um padrão de morfologia foliar, necessitando de equaçóes específicas para estimar a área foliar. Assim, o objetivo deste trabalho foi modelar e identificar os melhores modelos para a estimação da área foliar determinada por fotos digitais, de três híbridos de canola, em função do comprimento, ou da largura e/ou do produto comprimento vezes largura do limbo foliar.

\section{MATERIAL E MÉTOdOS}

Foram conduzidos três ensaios de uniformidade (experimentos sem tratamentos, em que a cultura agrícola e todos os procedimentos realizados durante o experimento são homogêneos em toda a área experimental) com a cultura de canola (Brassica napus L.) em uma área experimental de $45 \mathrm{~m} \times 60 \mathrm{~m}\left(2.700 \mathrm{~m}^{2}\right)$, localizada a $29^{\circ} 42^{\prime} \mathrm{S}, 53^{\circ} 49^{\prime} \mathrm{W}$ e a $95 \mathrm{~m}$ de altitude. Conforme classificação de Köppen, o clima da região é do tipo Cfa, subtropical úmido, com verōes quentes e sem estação seca definida (Heldwein et al., 2009). O solo é classificado como Argissolo Vermelho distrófico arênico (Santos et al., 2006).

Em cada ensaio de uniformidade, de $15 \mathrm{~m} \times 60 \mathrm{~m}$ $\left(900 \mathrm{~m}^{2}\right)$, foi avaliado um dos seguintes híbridos de canola: Hyola 61, Hyola 76 e Hyola 433. Nos três ensaios de uniformidade, a semeadura foi realizada em linhas, com espaçamento de $0,50 \mathrm{~m}$ entre linhas, no dia 13/6/2013, com emergência das plantas em 20/6/2013. A densidade, avaliada no florescimento da cultura, foi de 320.267, 351.067 e 410.800 plantas $\mathrm{ha}^{-1}$, respectivamente, para os híbridos Hyola 61, Hyola 76 e Hyola 433. A adubação de base foi de $8 \mathrm{~kg} \mathrm{ha}^{-1}$ de N, $80 \mathrm{~kg} \mathrm{ha}^{-1}$ de $\mathrm{P}_{2} \mathrm{O}_{5}$ e $80 \mathrm{~kg} \mathrm{ha}^{-1}$ de $\mathrm{K}_{2} \mathrm{O}$.

Em cada híbrido de canola (Hyola 61, Hyola 76 e Hyola 433), fizeram-se quatro coletas de 125 folhas cada. Essas coletas foram realizadas aos 77, 84, $91 \mathrm{e}$ 97 dias após a semeadura, totalizando 1.500 folhas (3 híbridos $\times 4$ coletas por híbrido $\times 125$ folhas por coleta). Coletaram-se de forma aleatória folhas expandidas dos terços inferior, médio e superior de cada planta. Essas amostras de folhas foram coletadas a fim de obter representatividade do dossel das plantas. Particularmente neste estudo, embora as mediçôes tenham sido feitas no tempo, foram em distintas plantas e folhas e, assim, consideradas como independentes. Adicionalmente, o foco do estudo foi construir modelos para estimar a área foliar de canola em função das dimensôes foliares, 
independentemente da fase de desenvolvimento da cultura. Em cada folha, mensuraram-se o comprimento (C) e a largura (L) do limbo foliar (Figura 1a,b,c) com régua milimetrada. Posteriormente, foi calculado o produto do comprimento vezes a largura $(\mathrm{C} \times \mathrm{L})$ do limbo foliar. Em seguida, determinou-se a área foliar real de cada uma das 1.500 folhas, por meio de fotos digitais. Para isso, cada folha foi colocada sob vidro transparente e, em seguida, fotografada com câmera digital da marca Casio, modelo Exilim EX-ZS5 $5^{\circ}$, disposta numa base perpendicular a $50 \mathrm{~cm}$ de distância em relação à folha, usando resolução de três megapixels. Essas 1.500 fotos (imagens) foram processadas, individualmente, com o programa Sigma Scan Pro v. $5.0^{\circledR}$ (Jandel Scientific, 1991) para a determinação da área foliar de cada folha, por meio do método de fotos digitais $(\mathrm{Y})$.

Para cada híbrido, de cada uma das quatro coletas, foram separadas aleatoriamente $80 \%$ das folhas (100 folhas) para a geração de modelos e $20 \%$ das folhas ( 25 folhas) para a validação dos modelos. Assim, para cada híbrido, 400 folhas
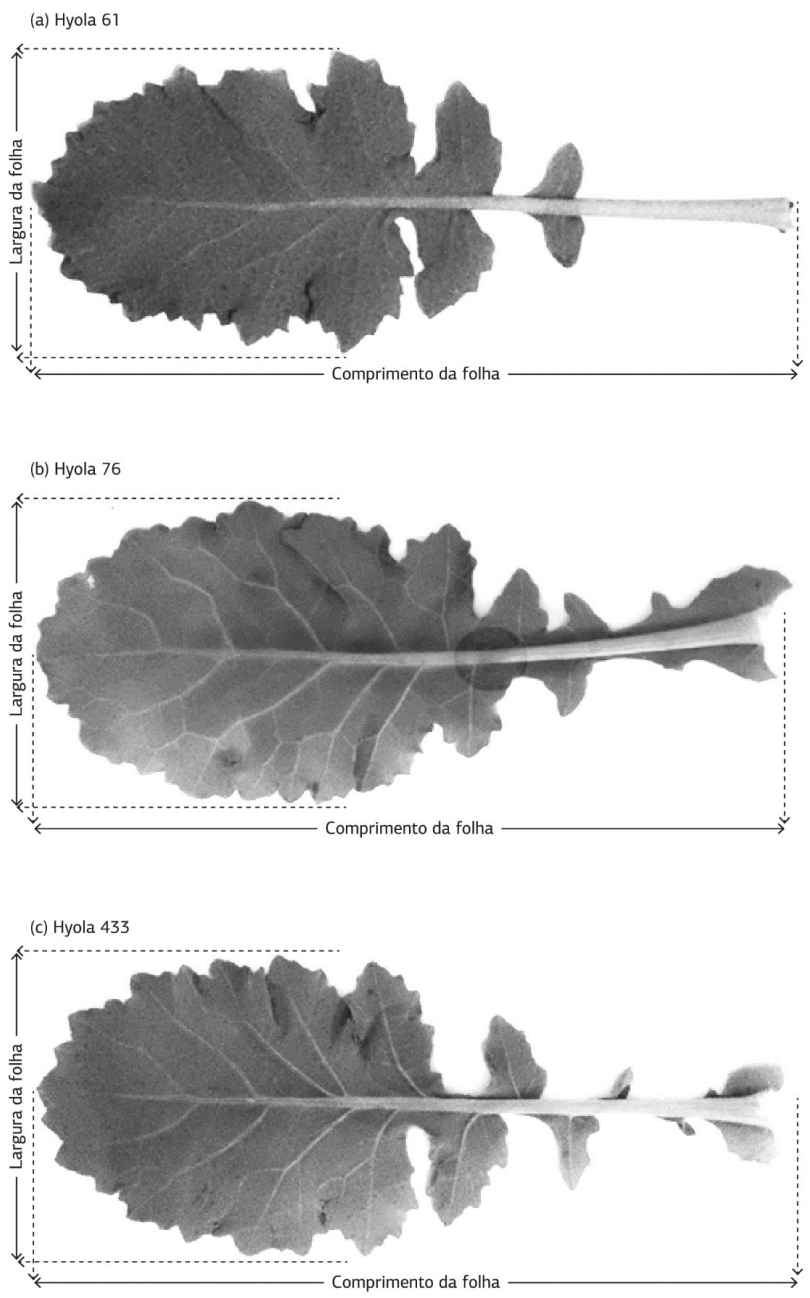

Figura 1. Comprimento e largura de uma folha de canola (Brassica napus L.), nos híbridos: (a) Hyola 61, (b) Hyola 76 e (c) Hyola 433. foram usadas para a geração de modelos de estimação da área foliar em função das dimensóes lineares, e 100 folhas para a validação desses modelos. A utilização das 500 folhas para a geração dos modelos poderia conferir maior abrangência, confiabilidade e, provavelmente, melhor ajustamento dos modelos. No entanto, é importante avaliar o desempenho dos modelos em conjunto de dados independentes, ou seja, náo nos mesmos dados que foram utilizados para a geraçáo dos modelos. Por isso, optou-se por dividir o conjunto de dados nas proporçóes de $80 \%$ e $20 \%$, respectivamente, para geração e validação dos modelos. Com essa divisão, e com as estatísticas de validação dos modelos descritas a seguir, é possível inferir sobre a qualidade do ajuste e, adicionalmente, avaliar se os modelos estimam adequadamente, superestimam ou subestimam a área foliar.

Para os dados do C, da L, do $\mathrm{C} \times \mathrm{L}$ e de $\mathrm{Y}$, de cada híbrido e conjunto de folhas ( 400 folhas para a geração e 100 folhas para validação dos modelos), obtiveram-se as estatísticas: mínimo, média, mediana, máximo, desviopadrão, coeficiente de variação, curtose e assimetria e, após foi verificada a normalidade, por meio do teste de Kolmogorov-Smirnov. Também, para cada híbrido, a partir das 400 folhas utilizadas para geraçấo dos modelos, foram feitos histogramas de frequência e gráficos de dispersão entre $\mathrm{C}, \mathrm{L}, \mathrm{C} \times \mathrm{L}$ e Y. Posteriormente, para cada híbrido, com as 400 folhas, modelou-se a área foliar determinada por fotos digitais $(\mathrm{Y})$ em funçáo do $\mathrm{C}$, ou da $\mathrm{L}$ e/ou do $\mathrm{C} \times \mathrm{L}$, por meio dos modelos: quadrático $\left(Y=a+b x+c x^{2}\right)$, potência $\left(Y=a x^{b}\right)$ e linear $(\mathrm{Y}=\mathrm{a}+\mathrm{bx})$, totalizando 27 equaçōes (três modelos $\times$ três variáveis independentes $\times$ três híbridos). Nesses modelos, $x$ representa a dimensão linear da folha $(\mathrm{C}, \mathrm{L}$ ou $\mathrm{C} \times \mathrm{L})$. $\mathrm{Na}$ prática, é importante gerar modelos com base em uma medida de dimensão linear da folha $(\mathrm{C}$ ou L $)$ em relação a duas medidas $(\mathrm{C} \times \mathrm{L})$, pela economia de trabalho com o uso de apenas uma dimensão da folha. Assim, optou-se por gerar modelos com as três variáveis preditoras da área foliar ( $\mathrm{C}, \mathrm{L}$ ou $\mathrm{C} \times \mathrm{L}$ ) testadas individualmente.

A validação dos nove modelos de estimação da área foliar foi realizada com base nos 100 valores estimados pelo modelo $\left(\hat{\mathrm{Y}}_{\mathrm{i}}\right)$ e os 100 valores observados $\left(\mathrm{Y}_{\mathrm{i}}\right)$. Em cada modelo, ajustou-se uma regressão linear simples $\left(\hat{\mathrm{Y}}_{\mathrm{i}}=\mathrm{a}+\mathrm{b} \mathrm{Y}_{\mathrm{i}}\right)$ da área foliar estimada pelo modelo (variável dependente), em função da área foliar observada (variável independente). Foram testadas as hipóteses $\mathrm{H}_{0}: \mathrm{a}=0$ versus $\mathrm{H}_{1}: \mathrm{a} \neq 0$ e $\mathrm{H}_{0}$ : $\mathrm{b}=1$ versus $\mathrm{H}_{1}: \mathrm{b} \neq 1$, por meio do teste t de Student a $5 \%$ de probabilidade. A interpretação desses testes de hipóteses possibilita inferir se os modelos estimam adequadamente, superestimam ou subestimam a área foliar. Os modelos mais adequados são aqueles em que o coeficiente linear (a) não difere de zero (reta passa pela origem) e o coeficiente angular (b) não difere de um (modelo estima adequadamente a área foliar), ou seja, nessa situação, os modelos não super ou subestimam a área foliar. 
A seguir, calcularam-se os coeficientes de correlação linear de Pearson $(r)$ e de determinação $\left(R^{2}\right)$ entre $\hat{Y}_{i}$ e $Y_{i}$. Ainda, para cada modelo, calculou-se o erro absoluto médio (EAM), a raiz do quadrado médio do erro (RQME) e o índice de Willmott (1981), conforme equaçôes descritas em Cargnelutti et al. (2012a, b) e Toebe et al. (2012), considerando, em cada híbrido, $\mathrm{n}=100$ folhas.

Para a escolha dos melhores modelos de estimação da área foliar de canola, em função do $\mathrm{C}$, ou da L e/ou do $\mathrm{C} \times \mathrm{L}$ do limbo foliar, foram usados os seguintes critérios: coeficiente linear não diferente de zero, coeficiente angular não diferente de um, coeficientes de correlação linear de Pearson e de determinação mais próximos de um, erro absoluto médio e raiz do quadrado médio do erro mais próximo de zero, e índice d de Willmott (1981) mais próximo de um. As análises estatísticas foram realizadas com auxílio do aplicativo Microsoft Office Excel $^{\bullet}$ e do software Statistica $7.0^{\circ}$ (Statsoft, 2005).

\section{RESULTADOS E DISCUSSÃO}

De maneira geral, nos conjuntos de dados de geração e de validação dos modelos para a estimação da área foliar, em média, o comprimento (C) do limbo foliar dos híbridos Hyola 61, Hyola 76 e Hyola 433 foi decrescente, nessa ordem. Já a largura (L) do limbo foliar, o produto do comprimento vezes a largura $(\mathrm{C} \times \mathrm{L})$ do limbo foliar e área foliar determinada por fotos digitais $(\mathrm{Y})$ foram decrescentes na seguinte ordem: Hyola 76, Hyola 61 e Hyola 433 (Tabela 1). Esses padróes distintos de $\mathrm{C}$ e $\mathrm{L}$, e consequentemente de $\mathrm{C} \times \mathrm{L}$ e $\mathrm{Y}$, entre os híbridos Hyola 61, Hyola 76 e Hyola 433, sugerem que a geração e a validação de modelos sejam específicas por híbrido.

Para os híbridos Hyola 61, Hyola 76 e Hyola 433, nos conjuntos de dados de geração e de validaçáo dos modelos, o coeficiente de variaçáo $(\mathrm{CV})$ do produto do comprimento vezes a largura $(\mathrm{C} \times \mathrm{L})$ do limbo foliar e da área foliar $(\mathrm{Y})$ foi, aproximadamente, o dobro do $\mathrm{CV}$ do comprimento (C) e da largura (L) do limbo foliar (Tabela 1). Em outros estudos, como os conduzidos nas culturas de mucuna-cinza (Cargnelutti et al., 2012a) e feijão-de-porco (Toebe et al., 2012), também verificou-se maior variabilidade relativa para o produto do comprimento vezes a largura e área foliar em relação ao comprimento e à largura. Já entre os híbridos, nos conjuntos de dados de geração e de validação dos modelos, o $\mathrm{CV}$ do $\mathrm{C}, \mathrm{L}, \mathrm{C} \times \mathrm{L}$ e $\mathrm{Y}$, foi decrescente na seguinte ordem: Hyola 76, Hyola 433 e Hyola 61. Esse distinto padrão de variabilidade entre os híbridos reforça a inferência de que a geração e a validação de modelos devem ser específicas por híbrido. A ampla variabilidade, garantida pela manutenção de valores extremos, é importante na modelagem e, consequentemente, na representatividade dos modelos.

Para o comprimento (C) e a largura (L) do limbo foliar do híbrido Hyola 61, nos conjuntos de dados de geraçáo e de validaçáo dos modelos, a curtose não diferente de três $(p>0,05)$, a assimetria não diferente de zero $(p>0,05)$, os elevados valores-p do teste normalidade de KolmogorovSmirnov ( $\mathrm{p} \geq 0,171)$, aliado à magnitude semelhante da média e da mediana, caracterizam bom ajuste à distribuição normal. Já o comprimento vezes a largura $(\mathrm{C} \times \mathrm{L})$ do limbo foliar e a área foliar $(\mathrm{Y})$ apresentaram leve afastamento da normalidade, caracterizado, principalmente, pela assimetria positiva (Tabela 1 e Figura 2).

Nos híbridos Hyola 76 e Hyola 433, nos conjuntos de dados de geração e de validação dos modelos, o comprimento (C) e a largura (L) do limbo foliar apresentaram menores coeficientes de curtose $(3,63 \leq$ curtose $\leq 6,04)$ e de assimetria $(0,59 \leq$ assimetria $\leq 1,45)$, quando comparados ao comprimento vezes a largura $(\mathrm{C} \times \mathrm{L})$ do limbo foliar e a área foliar $(\mathrm{Y})$, que apresentaram elevados coeficientes de curtose $(6,58 \leq$ curtose $\leq 16,52)$ e de assimetria positiva $(1,54 \leq$ assimetria $\leq 3,09)$. Portanto, pode-se inferir que $\mathrm{C}$ e $\mathrm{L}$ foliar apresentaram menor afastamento da normalidade, quando comparados $\mathrm{C} \times \mathrm{L}$ e $\mathrm{Y}$. Os valores elevados de $\mathrm{C}$, $\mathrm{L}, \mathrm{C} \times \mathrm{L}$ e $\mathrm{Y}$, mantidos no banco de dados, por refletirem condiçóes reais das folhas, explicam a assimetria positiva da distribuição, a maior magnitude da média em relação à mediana e os reduzidos valores-p do teste de normalidade de Kolmogorov-Smirnov ( $\mathrm{p}<0,171$ ) em 13 dos 16 casos (dois conjuntos de dados $\times$ dois híbridos $\times$ quatro variáveis) (Tabela 1 e Figuras 3 e 4). Assim, de maneira geral, nos três híbridos, o elevado número de folhas, a ampla variabilidade dos dados de $\mathrm{C}$ e L, e, consequentemente, $\mathrm{C} \times \mathrm{L}$ e Y, refletem condiçóes reais das folhas de canola e, portanto, caracterizam a adequabilidade desse conjunto de dados para a geração e validaçáo de modelos.

Visualiza-se nos gráficos de dispersão que nos híbridos Hyola 61, Hyola 76 e Hyola 433 há padróes de não linearidade entre $\mathrm{C}$ e $\mathrm{Y}$ e entre $\mathrm{L}$ e $\mathrm{Y}$, e lineariade entre $\mathrm{C} \times \mathrm{L}$ eY (Figuras 2, 3 e 4), o que sugere melhor ajuste de modelos não lineares e lineares, respectivamente. Esses padrôes também foram verificados em couve-folha (Marcolini et al., 2005), pinhão-manso (Severino et al., 2007), feijão-caupi (Lima et al., 2008), girassol (Aquino et al., 2011), feijão-deporco (Toebe et al., 2012), mucuna-cinza (Cargnelutti et al., 2012a) e nabo-forrageiro (Cargnelutti et al., 2012b). Essa constatação é visual e, por isso, deve-se investigar, por meio de procedimentos estatísticos, os modelos que melhor se ajustam em cada conjunto de dados de geração e validar os modelos com conjunto de dados independentes.

$\mathrm{O}$ coeficiente de determinação $\left(\mathrm{R}^{2}\right)$ das equaçôes para modelar a área foliar, dos híbridos Hyola 61, Hyola 76 e Hyola 433, determinada por fotos digitais $(\mathrm{Y})$ em função do 
$\mathrm{C}$, ou da $\mathrm{L}$ e/ou do $\mathrm{C} \times \mathrm{L}$, por meio dos modelos quadrático $\left(\mathrm{Y}=\mathrm{a}+\mathrm{bx}+\mathrm{cx}^{2}\right)$, potência $\left(\mathrm{Y}=\mathrm{ax} \mathrm{x}^{\mathrm{b}}\right)$ e linear $(\mathrm{Y}=\mathrm{a}+\mathrm{bx})$, oscilou entre 0,8734 e 0,9789 (Tabela 2). Os modelos, gerados nesse estudo, apresentaram ajuste superior aos modelos estabelecidos por Chavarria et al. (2011), para o híbrido de canola Hyola 61, cujo maior $\mathrm{R}^{2}$ foi 0,7704 , para o modelo quadrático. Nesse estudo, num primeiro momento, pode-se inferir que os elevados valores do coeficiente de determinaçáo

Tabela 1. Número de folhas (n), mínimo, média, mediana, máximo, desvio-padrão, coeficiente de variação $(\mathrm{CV})$, curtose, assimetria e valor-p do teste de Kolmogorov-Smirnov para o comprimento (C), a largura (L), o produto comprimento vezes largura $(\mathrm{C} \times \mathrm{L})$ do limbo foliar e a área foliar determinada por fotos digitais (Y), em três híbridos de canola (Brassica napus L.), nos conjuntos de dados de geração e de validação dos modelos

\begin{tabular}{|c|c|c|c|c|c|c|}
\hline \multirow[t]{2}{*}{ Estatística } & \multicolumn{3}{|c|}{ Geração dos modelos } & \multicolumn{3}{|c|}{ Validação dos modelos } \\
\hline & Hyola 61 & Hyola 76 & Hyola 433 & Hyola 61 & Hyola 76 & Hyola 433 \\
\hline & \multicolumn{6}{|c|}{ C - comprimento do limbo foliar, em cm } \\
\hline$n$ & 400 & 400 & 400 & 100 & 100 & 100 \\
\hline Mínimo & 5,70 & 4,10 & 3,70 & 5,50 & 3,40 & 4,20 \\
\hline Média & 16,02 & 15,09 & 14,04 & 15,87 & 14,54 & 14,03 \\
\hline Mediana & 16,00 & 13,45 & 12,90 & 15,85 & 12,70 & 13,45 \\
\hline Máximo & 26,10 & 42,10 & 35,10 & 24,70 & 45,00 & 31,60 \\
\hline Desvio-padrão & 4,20 & 6,91 & 5,66 & 3,88 & 7,27 & 5,23 \\
\hline $\mathrm{CV}(\%)$ & 26,20 & 45,78 & 40,32 & 24,45 & 50,00 & 37,28 \\
\hline Curtose & $2,55^{\mathrm{ns}}$ & $4,67^{*}$ & $4,20^{*}$ & $3,09^{\text {ns }}$ & $5,64^{*}$ & $3,63^{\text {ns }}$ \\
\hline Assimetria & $-0,04^{\text {ns }}$ & $1,23^{*}$ & $1,00^{*}$ & $-0,13^{\text {ns }}$ & $1,37^{*}$ & $0,73^{*}$ \\
\hline \multirow[t]{2}{*}{ Valor-p } & 0,662 & 0,000 & 0,003 & 0,928 & 0,011 & 0,364 \\
\hline & \multicolumn{6}{|c|}{ L - Largura do limbo foliar, em cm } \\
\hline$n$ & 400 & 400 & 400 & 100 & 100 & 100 \\
\hline Mínimo & 2,00 & 2,30 & 1,80 & 2,40 & 1,90 & 1,90 \\
\hline Média & 6,51 & 6,81 & 5,94 & 6,47 & 6,52 & 5,85 \\
\hline Mediana & 6,40 & 6,30 & 5,55 & 6,40 & 5,70 & 5,90 \\
\hline Máximo & 10,50 & 17,30 & 14,00 & 9,50 & 18,40 & 12,80 \\
\hline Desvio-padrão & 1,58 & 2,55 & 2,15 & 1,44 & 2,81 & 1,97 \\
\hline CV(\%) & 24,24 & 37,43 & 36,15 & 22,28 & 43,08 & 33,78 \\
\hline Curtose & $2,74^{\mathrm{ns}}$ & $4,39^{*}$ & $3,88^{*}$ & $2,77^{\text {ns }}$ & $6,04^{*}$ & $3,69^{\text {ns }}$ \\
\hline Assimetria & $0,00^{\text {ns }}$ & $1,10^{*}$ & $0,86^{*}$ & $-0,10^{\text {ns }}$ & $1,45^{*}$ & $0,59^{*}$ \\
\hline \multirow[t]{2}{*}{ Valor-p } & 0,171 & 0,001 & 0,001 & 0,999 & 0,053 & 0,683 \\
\hline & \multicolumn{6}{|c|}{$\mathrm{C} \times \mathrm{L}-$ produto do comprimento vezes a largura do limbo foliar, em $\mathrm{cm}^{2}$} \\
\hline$n$ & 400 & 400 & 400 & 100 & 100 & 100 \\
\hline Mínimo & 11,40 & 9,43 & 7,38 & 13,20 & 6,46 & 8,40 \\
\hline Média & 110,51 & 119,78 & 95,01 & 107,88 & 114,54 & 91,70 \\
\hline Mediana & 101,74 & 86,59 & 72,00 & 101,69 & 71,88 & 81,58 \\
\hline Máximo & 268,83 & 726,60 & 487,20 & 231,24 & 828,00 & 369,92 \\
\hline Desvio-padrão & 52,13 & 107,68 & 75,74 & 47,11 & 120,31 & 64,52 \\
\hline $\mathrm{CV}(\%)$ & 47,17 & 89,89 & 79,72 & 43,67 & 105,04 & 70,36 \\
\hline Curtose & $2,74^{\mathrm{ns}}$ & $9,44^{*}$ & $8,15^{*}$ & $2,92^{\text {ns }}$ & $16,52^{*}$ & $6,58^{*}$ \\
\hline Assimetria & $0,53^{*}$ & $2,28^{*}$ & $1,99 *$ & $0,49^{*}$ & $3,09^{*}$ & $1,59^{*}$ \\
\hline \multirow[t]{2}{*}{ Valor-p } & 0,022 & 0,000 & 0,000 & 0,600 & 0,000 & 0,060 \\
\hline & \multicolumn{6}{|c|}{$\mathrm{Y}$ - área foliar, em $\mathrm{cm}^{2}$} \\
\hline$n$ & 400 & 400 & 400 & 100 & 100 & 100 \\
\hline Mínimo & 6,82 & 6,33 & 4,42 & 9,13 & 4,40 & 5,26 \\
\hline Média & 51,65 & 61,59 & 47,82 & 51,01 & 59,05 & 45,65 \\
\hline Mediana & 47,71 & 44,42 & 36,44 & 48,01 & 39,50 & 40,61 \\
\hline Máximo & 137,48 & 328,40 & 228,67 & 106,13 & 402,31 & 181,32 \\
\hline Desvio-padrão & 24,27 & 53,50 & 36,20 & 20,98 & 59,50 & 29,05 \\
\hline $\mathrm{CV}(\%)$ & 46,98 & 86,86 & 75,70 & 41,13 & 100,77 & 63,64 \\
\hline Curtose & $3,11^{\mathrm{ns}}$ & $8,29^{*}$ & $8,31^{*}$ & $2,87^{\text {ns }}$ & $15,84^{*}$ & $7,00^{*}$ \\
\hline Assimetria & $0,67^{*}$ & $2,18^{*}$ & $2,01^{*}$ & $0,42^{*}$ & $3,06^{*}$ & $1,54^{*}$ \\
\hline Valor-p & 0,012 & 0,000 & 0,000 & 0,775 & 0,001 & 0,255 \\
\hline
\end{tabular}

* Curtose difere de três ou assimetria difere de zero, pelo teste t, em nível de $5 \%$ de probabilidade. ${ }^{\text {ns }}$ Năo significativo. 

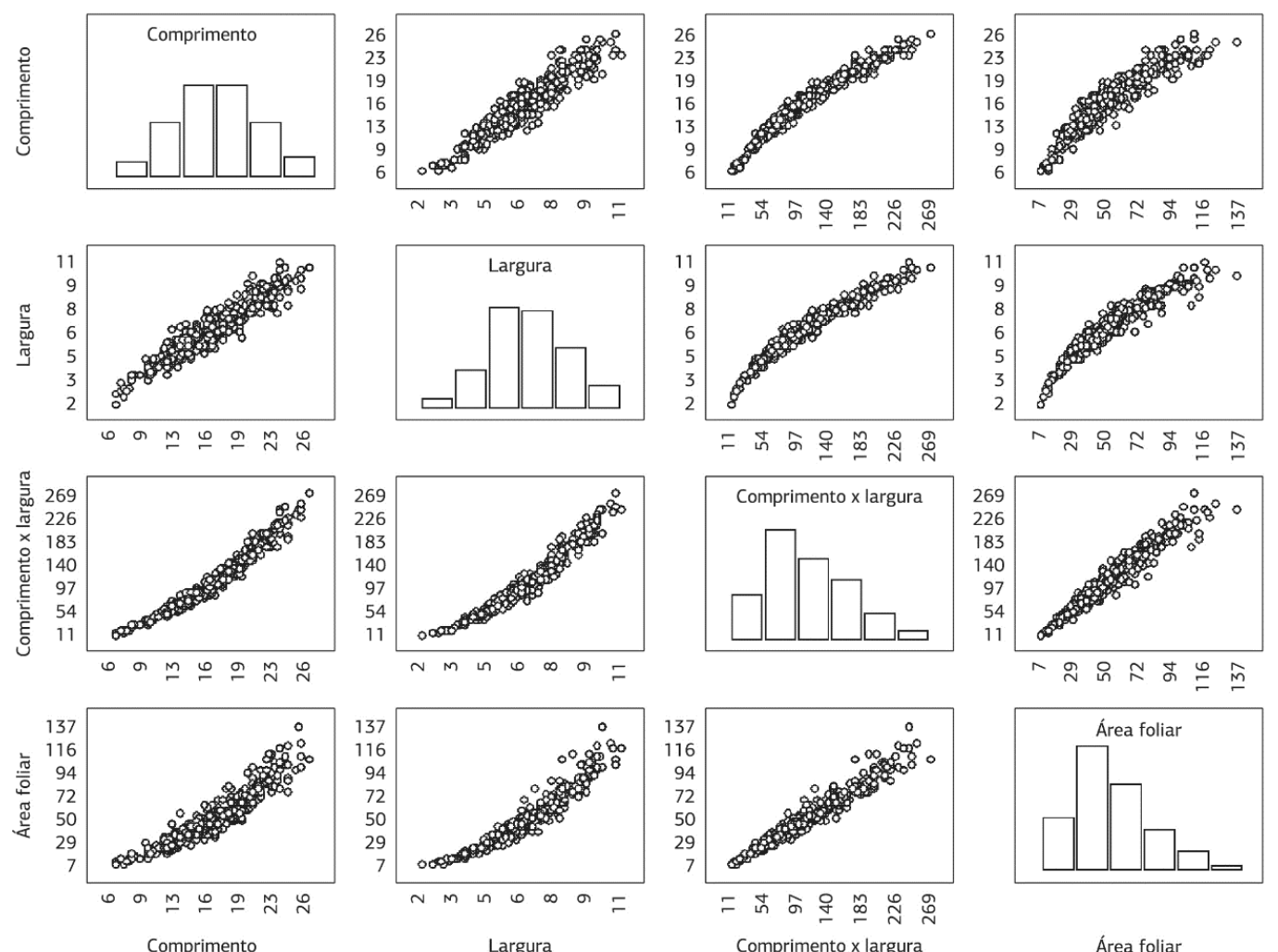

Figura 2. Matriz com o histograma de frequência (na diagonal) e gráficos de dispersão entre o comprimento, em cm, a largura, em cm, o produto comprimento $\times$ largura, em $\mathrm{cm}^{2}$, e a área foliar determinada por fotos digitais, em $\mathrm{cm}^{2}$ de 400 folhas do híbrido de canola (Brassica napus L.), Hyola 61.
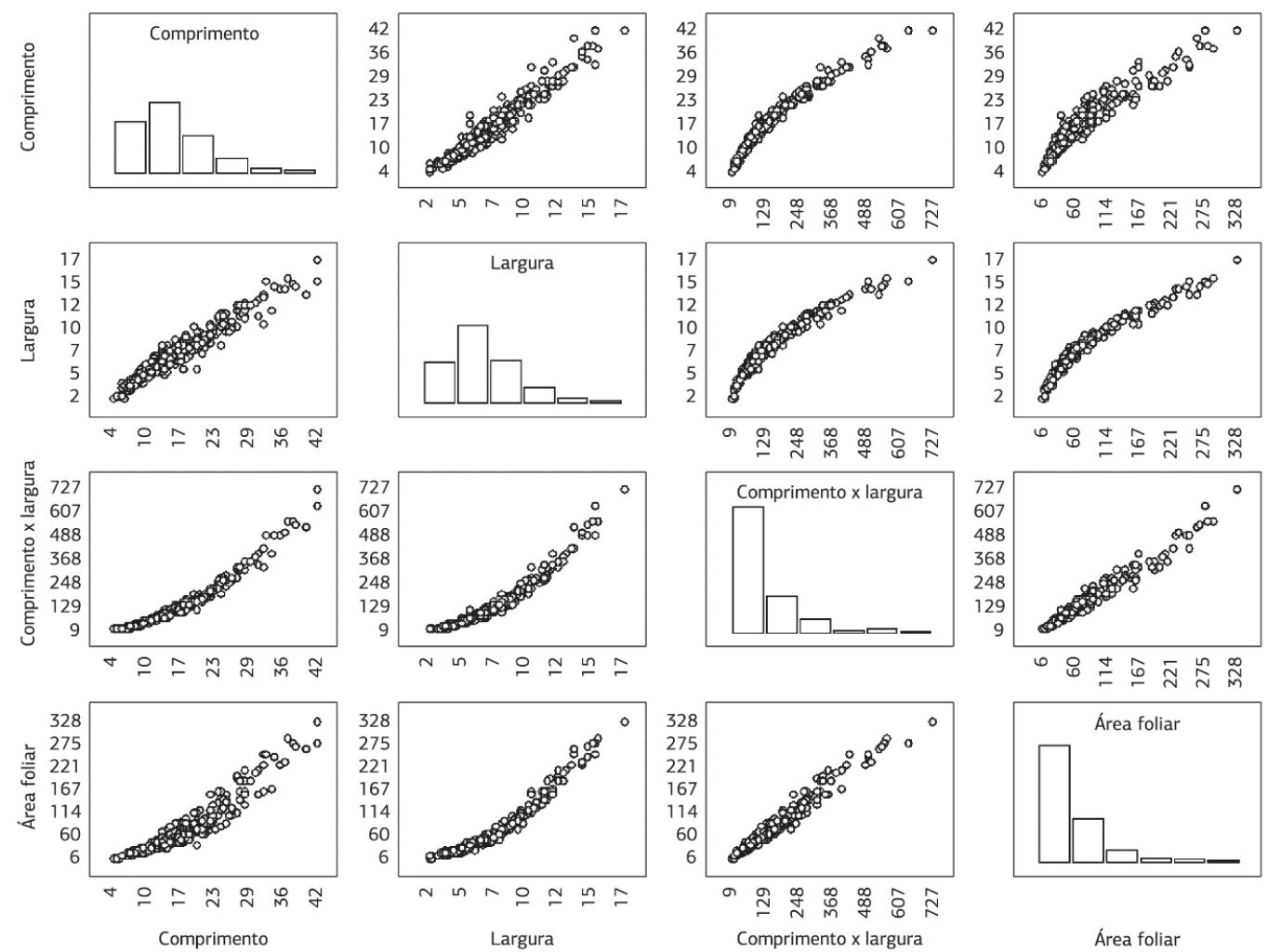

Área foliar

Figura 3. Matriz com o histograma de frequência (na diagonal) e gráficos de dispersão entre o comprimento, em cm, a largura, em cm, o produto comprimento $\times$ largura, $\mathrm{em}^{2}$, e a área foliar determinada por fotos digitais, em $\mathrm{cm}^{2}$ de 400 folhas do híbrido de canola (Brassica napus L.), Hyola 76. 

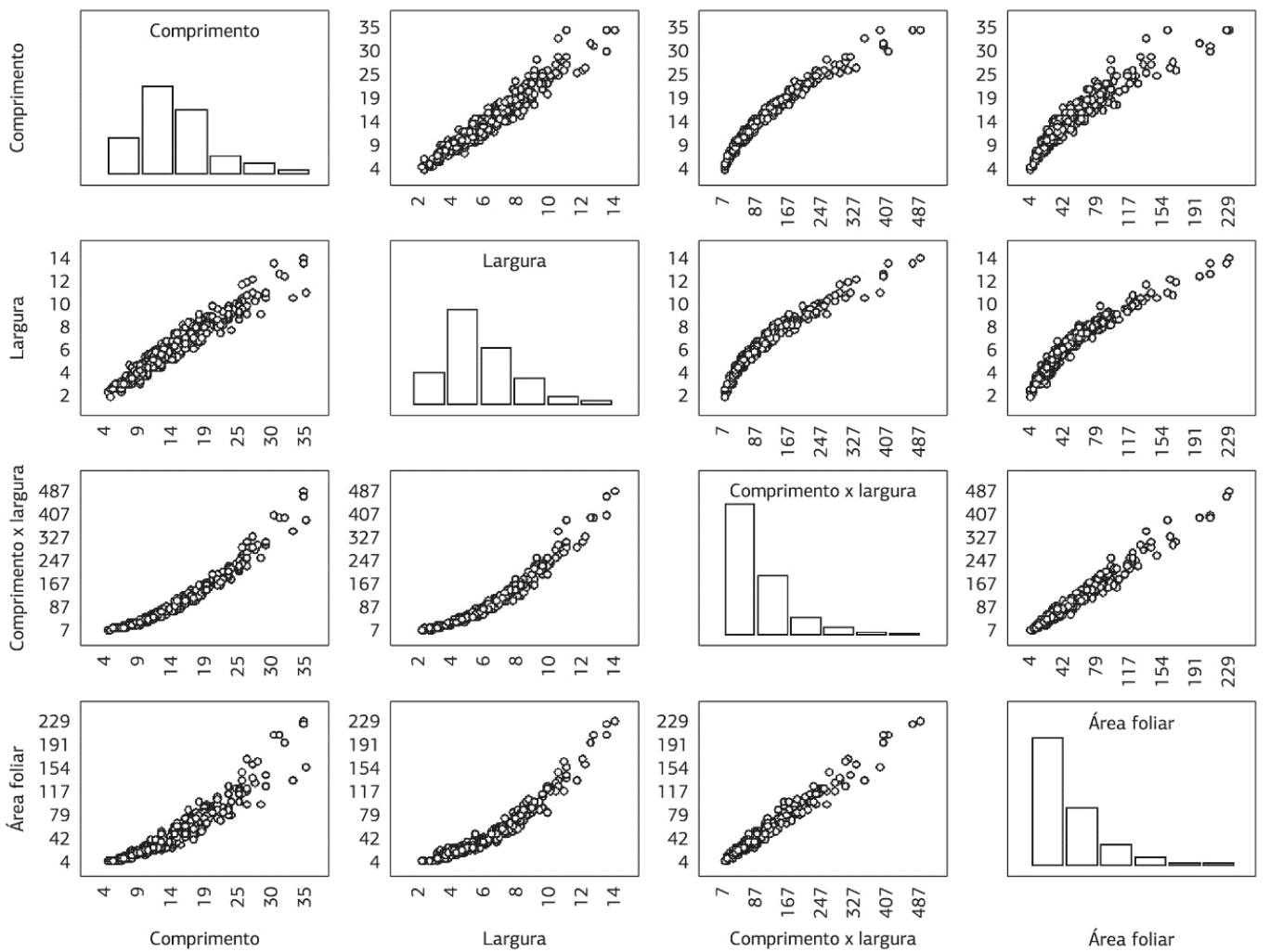

Figura 4. Matriz com o histograma de frequência (na diagonal) e gráficos de dispersão entre o comprimento, em $\mathrm{cm}$, a largura, em cm, o produto comprimento $\times$ largura, em $\mathrm{cm}^{2}$, e a área foliar determinada por fotos digitais, em $\mathrm{cm}^{2}$ de 400 folhas do híbrido de canola (Brassica napus L.), Hyola 433.

$\left(\mathrm{R}^{2} \geq 0,8734\right)$ sugerem que todos os modelos poderiam ser utilizados para estimar a área foliar. Porém, a variabilidade existente entre os ajustes desses 27 modelos $\left(0,8734 \leq \mathrm{R}^{2}\right.$ $\leq 0,9789)$ possibilita a investigação de qual seria o melhor modelo para cada híbrido.

Para os híbridos de canola Hyola 61, Hyola 76 e Hyola 433, os modelos quadrático, potência e linear, para a estimação da área foliar (Y) com base no comprimento (C) do limbo foliar, apresentaram ajuste inferior (menores valores de $\mathrm{R}^{2}$ ), quando comparados aos modelos gerados com base em L e C $\times$ L (Tabela 2). Na cultura do girassol, modelos gerados para estimativa da área foliar a partir da largura foram mais precisos do que os gerados com base no comprimento (Maldaner et al., 2009; Aquino et al., 2011). Para a estimação da área foliar de pinhão-manso, Severino et al. (2007) também verificaram que o modelo gerado com base na largura da folha apresentou elevado coeficiente de determinação $\left(\mathrm{R}^{2}=0,97\right)$. Já os modelos gerados com base em $\mathrm{C} \times \mathrm{L}$ foram recomendados para estimar a área foliar nas culturas de couve-folha (Marcolini et al., 2005), feijão-caupi (Lima et al., 2008), batata aos 50 dias após a emergência (Busato et al., 2010) e nabo-forrageiro (Cargnelutti et al., 2012b). Assim, pode-se inferir que, para os três híbridos de canola, somente a medida do C não é adequada para ser utilizada no modelo de estimação da área foliar. Assim, é importante investigar, entre a $\mathrm{L}$ e o $\mathrm{C} \times \mathrm{L}$, qual é a melhor variável preditora da área foliar. Nessa investigação, deve-se considerar que, para inferências com base em L, é necessário apenas uma dimensão foliar (largura da folha), enquanto com base em $\mathrm{C} \times \mathrm{L}$ o número de mediçóes é o dobro (largura e comprimento da folha).

Para os três híbridos, o modelo linear, com base no $\mathrm{C} \times \mathrm{L}$, apresentou melhor ajuste $\left(0,9449 \leq \mathrm{R}^{2} \leq 0,9691\right)$, comparado aos ajustes obtidos a partir de $\mathrm{L}\left(0,9108 \leq \mathrm{R}^{2}\right.$ $\leq 0,9217)$ e de $C\left(0,8734 \leq R^{2} \leq 0,8895\right)$ (Tabela 2$)$. Esse fato confirma os padróes de linearidade entre $\mathrm{C} \times \mathrm{L}$ e $\mathrm{Y}$ e de não linearidade entre $\mathrm{C}$ e $\mathrm{Y}$ e $\mathrm{L}$ e $\mathrm{Y}$, visualizados nos gráficos de dispersão (Figuras 2, 3 e 4). Portanto, com a opção do modelo linear, pela simplicidade em relação ao quadrático e potência, a variável $\mathrm{C} \times \mathrm{L}$ deveria ser utilizada como preditora da área foliar. Porém, ajustes elevados também foram obtidos para os três híbridos nos modelos quadrático e potência gerados a partir de $\mathrm{L}\left(0,9316 \leq \mathrm{R}^{2} \leq 0,9789\right) \mathrm{e}$ $\mathrm{C} \times \mathrm{L}\left(0,9451 \leq \mathrm{R}^{2} \leq 0,9708\right)$. Portanto, na prática, diante do trabalho duplicado necessário para a obtenção do $\mathrm{C} \times \mathrm{L}$ (duas medidas) em relaçáo a L (uma medida), aliado aos elevados escores de $\mathrm{R}^{2}$, é adequado utilizar os modelos 
Tabela 2. Modelos para a determinação da área foliar obtida por fotos digitais (Y), utilizando o comprimento (C), a largura (L) e o produto comprimento vezes largura $(\mathrm{C} \times \mathrm{L})$ do limbo foliar como variáveis independentes $(\mathrm{x})$ e o coeficiente de determinação $\left(\mathrm{R}^{2}\right)$ de cada modelo, com base em 400 folhas de cada híbrido de canola (Brassica napus L.)

\begin{tabular}{|c|c|c|c|}
\hline Modelo & $\begin{array}{c}\text { Variável } \\
\text { independente }(x)\end{array}$ & Equação & Coeficiente de determinação \\
\hline & & Hyola 61 & \\
\hline 1) Quadrático & C & $\hat{Y}=3,9265+0,1660 x+0,1644 x^{2}$ & 0,8953 \\
\hline 2) Quadrático & L & $\hat{Y}=-1,1711+0,7324 x+1,0704 x^{2}$ & 0,9316 \\
\hline 3) Quadrático & $C \times L$ & $\hat{Y}=2,9200+0,4272 x+0,0001 x^{2}$ & 0,9451 \\
\hline 4) Potência & C & $\hat{Y}=0,3943 x^{1,7377}$ & 0,9186 \\
\hline 5) Potência & $\mathrm{L}$ & $\hat{Y}=1,3000 x^{1,9336}$ & 0,9531 \\
\hline 6) Potência & $C \times L$ & $\hat{Y}=0,6189 x^{0,9401}$ & 0,9603 \\
\hline 7) Linear & C & $\hat{Y}=-34,9179+5,4048 x$ & 0,8734 \\
\hline 8) Linear & $\mathrm{L}$ & $\hat{Y}=-43,8895+14,6716 x$ & 0,9108 \\
\hline \multirow[t]{2}{*}{ 9) Linear } & $C \times L$ & $\hat{Y}=1,6467+0,4525 x$ & 0,9449 \\
\hline & & Hyola 76 & \\
\hline 1) Quadrático & C & $\hat{Y}=-2,0169+1,4464 x+0,1517 x^{2}$ & 0,9288 \\
\hline 2) Quadrático & L & $\hat{Y}=3,5219-1,9074 x+1,3440 x^{2}$ & 0,9789 \\
\hline 3) Quadrático & $C \times L$ & $\hat{Y}=1,1296+0,5173 x-0,0001 x^{2}$ & 0,9696 \\
\hline 4) Potência & C & $\hat{Y}=0,6123 x^{1,6517}$ & 0,9389 \\
\hline 5) Potência & $\mathrm{L}$ & $\hat{Y}=1,0579 x^{2,0383}$ & 0,9733 \\
\hline 6) Potência & $C \times L$ & $\hat{Y}=0,7289 x^{0,9281}$ & 0,9708 \\
\hline 7) Linear & C & $\hat{Y}=-48,6371+7,3031 x$ & 0,8895 \\
\hline 8) Linear & L & $\hat{Y}=-75,6346+20,1473 x$ & 0,9217 \\
\hline \multirow[t]{2}{*}{ 9) Linear } & $C \times L$ & $\hat{Y}=3,0011+0,4891 x$ & 0,9691 \\
\hline & & Hyola 433 & \\
\hline 1) Quadrático & C & $\hat{Y}=-4,2239+1,5362 x+0,1330 x^{2}$ & 0,9172 \\
\hline 2) Quadrático & L & $\hat{Y}=5,3118-1,4727 x+1,2868 x^{2}$ & 0,9749 \\
\hline 3) Quadrático & $C \times L$ & $\hat{Y}=3,1224+0,4706 x-0,000001 x^{2}$ & 0,9681 \\
\hline 4) Potência & C & $\hat{Y}=0,4736 x^{1,7060}$ & 0,9412 \\
\hline 5) Potência & L & $\hat{Y}=1,4154 x^{1,9096}$ & 0,9613 \\
\hline 6) Potência & $C \times L$ & $\hat{Y}=0,7425 x^{0,9167}$ & 0,9672 \\
\hline 7) Linear & C & $\hat{Y}=-36,7545+6,0228 x$ & 0,8871 \\
\hline 8) Linear & L & $\hat{Y}=-48,1387+16,1664 x$ & 0,9183 \\
\hline 9) Linear & $C \times L$ & $\hat{Y}=3,1382+0,4703 x$ & 0,9681 \\
\hline
\end{tabular}

gerados em função de L, pela economia de trabalho com o uso de apenas uma dimensão da folha. Assim, para o híbrido Hyola 61, o modelo potência, com base em L, apresentou melhor ajuste $\left(\mathrm{R}^{2}=0,9531\right)$, comparado ao modelo quadrático $\left(\mathrm{R}^{2}=0,9316\right)$. Já para os híbridos Hyola 76 e Hyola 433, o modelo quadrático apresentou leve superioridade em relação ao modelo potência. Em videira, Borghezan et al. (2010), avaliando variedades de videira a campo, observaram que os modelos foram diferentes conforme a variedade, sendo que para a variedade Merlot, o melhor modelo foi o do tipo quadrático, e o modelo do tipo potência foi o mais adequado para as variedades Cabernet Sauvignon e Sauvignon Blanc.

Nos três híbridos, os indicadores da qualidade de ajuste dos modelos de estimação da área foliar de canola foram, de maneira geral, favoráveis ao modelo potência gerado em função da L, quando comparado ao modelo quadrático gerado em função da L (Tabela 3). Portanto, na prática, os modelos, do tipo potência, para os híbridos Hyola $61\left(\hat{Y}=1,3000 x^{1,9336}\right.$,
$\left.\mathrm{R}^{2}=0,9531\right)$, Hyola $76\left(\hat{\mathrm{Y}}=1,0579 \mathrm{x}^{2,0383}, \mathrm{R}^{2}=0,9733\right)$ e Hyola $433\left(\hat{Y}=1,4154 x^{1,9096}, R^{2}=0,9613\right)$, podem ser utilizados na estimação da área foliar $(\mathrm{Y})$ de canola com base em $x$, que representa largura do limbo foliar.

Estudos de modelagem de área foliar em função de dimensôes lineares das folhas foram desenvolvidos em outras culturas da família Brassicaceae por Toebe et al. (2010) e Cargnelutti et al. (2012b). De acordo com Cargnelutti et al. (2012b), o modelo potência, em função do produto C×L, é adequado para estimar a área foliar de nabo-forrageiro, o qual apresentou o mais elevado coeficiente de determinação $\left(\mathrm{R}^{2}=0,9862\right)$. Em crambe, Toebe et al. (2010) verificaram que a largura da folha é variável independente que permite a melhor estimativa de área foliar, quando apenas uma variável é avaliada, com destaque aos modelos quadrático e geométrico. Os autores verificaram também que, quando se considera o produto $\mathrm{C} \times \mathrm{L}$, os melhores modelos são do tipo linear. 
Tabela 3. Variáveis independentes (x) e coeficientes linear (a), angular (b), de correlaçáo linear de Pearson (r) e de determinação ( $\left.\mathrm{R}^{2}\right)$, obtidos na regressão linear ajustada entre a área foliar estimada (variável dependente) e a observada (variável independente). Erro absoluto médio (EAM), raiz do quadrado médio do erro (RQME), índice d de Willmott (1981) calculados com base nas áreas foliares estimadas e observadas, de 100 folhas de cada híbrido de canola (Brassica napus L.)

\begin{tabular}{|c|c|c|c|c|c|c|c|c|}
\hline Modelo & $x^{(1)}$ & a & b & $\mathbf{r}$ & $\mathbf{R}^{2}$ & EAM & RQME & d \\
\hline \multicolumn{9}{|c|}{ Hyola 61} \\
\hline 1) Quadrático & C & $2,8892^{\text {ns }}$ & $0,9319^{\text {ns }}$ & $0,9368^{*}$ & 0,8777 & 5,4659 & 7,4190 & 0,9674 \\
\hline 2) Quadrático & L & $1,3973^{\text {ns }}$ & $0,9639^{\text {ns }}$ & $0,9633^{*}$ & 0,9280 & 4,2698 & 5,6694 & 0,9812 \\
\hline 3) Quadrático & $C \times L$ & $0,5271^{\mathrm{ns}}$ & $0,9781^{\text {ns }}$ & $0,9646^{*}$ & 0,9306 & 4,0851 & 5,6233 & 0,9818 \\
\hline 4) Potência & C & $3,7671^{*}$ & $0,9054^{*}$ & $0,9380^{*}$ & 0,8800 & 5,4056 & 7,3283 & 0,9672 \\
\hline 5) Potência & L & $1,8288^{\text {ns }}$ & $0,9483^{\text {ns }}$ & $0,9633^{*}$ & 0,9281 & 4,2516 & 5,6705 & 0,9809 \\
\hline 6) Potência & $C \times L$ & $1,5743^{\text {ns }}$ & $0,9528^{\text {ns }}$ & $0,9656^{*}$ & 0,9324 & 4,0580 & 5,5076 & 0,9820 \\
\hline 7) Linear & C & $3,3403^{\text {ns }}$ & $0,9316^{\mathrm{ns}}$ & $0,9319^{*}$ & 0,8685 & 5,9482 & 7,7010 & 0,9649 \\
\hline 8) Linear & L & $1,7954^{\text {ns }}$ & $0,9651^{\mathrm{ns}}$ & $0,9574^{*}$ & 0,9166 & 4,8026 & 6,1183 & 0,9782 \\
\hline 9) Linear & $C \times L$ & $0,4346^{\text {ns }}$ & $0,9807^{\text {ns }}$ & $0,9651^{*}$ & 0,9315 & 4,0989 & 5,5886 & 0,9820 \\
\hline \multicolumn{9}{|c|}{ Hyola 76} \\
\hline 1) Quadrático & C & $6,1290^{*}$ & $0,8960^{*}$ & $0,9710^{*}$ & 0,9428 & 8,7776 & 14,4358 & 0,9836 \\
\hline 2) Quadrático & L & $-1,9697^{*}$ & $1,0273^{*}$ & $0,9940^{*}$ & 0,9880 & 4,5514 & 6,8783 & 0,9967 \\
\hline 3) Quadrático & $C \times L$ & $1,8573^{\text {ns }}$ & $0,9639^{*}$ & $0,9871^{*}$ & 0,9744 & 6,1017 & 9,4812 & 0,9933 \\
\hline 4) Potência & C & $8,7584^{*}$ & $0,8246^{*}$ & $0,9672^{*}$ & 0,9355 & 9,3217 & 16,5692 & 0,9768 \\
\hline 5) Potência & L & $0,1658^{\text {ns }}$ & $0,9752^{\text {ns }}$ & $0,9940^{*}$ & 0,9882 & 4,3727 & 6,6007 & 0,9968 \\
\hline 6) Potência & $C \times L$ & $3,7756^{*}$ & $0,9161^{*}$ & $0,9868^{*}$ & 0,9739 & 5,9474 & 10,2345 & 0,9919 \\
\hline 7) Linear & C & $8,5319^{*}$ & $0,8305^{*}$ & $0,9306^{*}$ & 0,8660 & 14,3280 & 21,8332 & 0,9607 \\
\hline 8) Linear & L & $2,0360^{\text {ns }}$ & $0,9088^{*}$ & $0,9557^{*}$ & 0,9135 & 11,3991 & 17,7300 & 0,9759 \\
\hline 9) Linear & $C \times L$ & $1,3419^{\text {ns }}$ & $0,9767^{\text {ns }}$ & $0,9877^{*}$ & 0,9756 & 5,8389 & 9,2464 & 0,9937 \\
\hline \multicolumn{9}{|c|}{ Hyola 433} \\
\hline 1) Quadrático & C & $2,6449^{\text {ns }}$ & $0,9742^{\text {ns }}$ & $0,9247^{*}$ & 0,8550 & 7,3741 & 11,7099 & 0,9597 \\
\hline 2) Quadrático & L & $-1,8819^{*}$ & $1,0409^{*}$ & $0,9877^{*}$ & 0,9755 & 3,1298 & 4,9081 & 0,9931 \\
\hline 3) Quadrático & $C \times L$ & $-0,2367^{\mathrm{ns}}$ & $1,0186^{\text {ns }}$ & $0,9752^{*}$ & 0,9510 & 4,5323 & 6,7282 & 0,9868 \\
\hline 4) Potência & C & $2,9737^{\mathrm{ns}}$ & $0,9513^{\text {ns }}$ & $0,9254^{*}$ & 0,8564 & 7,1852 & 11,3713 & 0,9610 \\
\hline 5) Potência & L & $-0,4967^{\mathrm{ns}}$ & $1,0023^{\text {ns }}$ & $0,9891^{*}$ & 0,9784 & 2,9393 & 4,3226 & 0,9944 \\
\hline 6) Potência & $C \times L$ & $0,6796^{\mathrm{ns}}$ & $0,9916^{\text {ns }}$ & $0,9758^{*}$ & 0,9522 & 4,4283 & 6,4285 & 0,9876 \\
\hline 7) Linear & C & $1,9456^{\text {ns }}$ & $1,0036^{\text {ns }}$ & $0,9253^{*}$ & 0,8562 & 8,9368 & 12,0743 & 0,9585 \\
\hline 8) Linear & L & $-2,3443^{\text {ns }}$ & $1,0668^{*}$ & $0,9708^{*}$ & 0,9426 & 6,3537 & 7,8816 & 0,9829 \\
\hline 9) Linear & $C \times L$ & $-0,2324^{\mathrm{ns}}$ & $1,0185^{\text {ns }}$ & $0,9752^{*}$ & 0,9510 & 4,5317 & 6,7278 & 0,9868 \\
\hline
\end{tabular}

\section{CONCLUSÃO}

Em canola, os modelos do tipo potência, para os híbridos Hyola $61\left(\hat{Y}=1,3000 x^{1,9336}, R^{2}=0,9531\right)$, Hyola 76 $\left(\hat{\mathrm{Y}}=1,0579 \mathrm{x}^{2,0383}, \mathrm{R}^{2}=0,9733\right)$ e Hyola $433\left(\hat{\mathrm{Y}}=1,4154 \mathrm{x}^{1,9096}\right.$, $\left.\mathrm{R}^{2}=0,9613\right)$, são adequados para a estimação da área foliar determinada por fotos digitais $(\mathrm{Y})$ em função da largura do limbo foliar (x).

\section{AGRADECIMENTOS}

Ao Conselho Nacional de Desenvolvimento Científico e Tecnológico (CNPq) e à Coordenação de Aperfeiçoamento de Pessoal de Nível Superior (CAPES), pelas bolsas concedidas. Ao pesquisador da Embrapa, Gilberto Omar Tomm, pela doação das sementes de canola (Brassica napus L.).

\section{REFERÊNCIAS}

Aquino, L. A., Santos, V. C., Jr., Guerra, J. V. S., \& Costa, M. M. (2011). Estimativa da área foliar do girassol por método não destrutivo. Bragantia, 70, 832-836. http://dx.doi.org/10.1590/ S0006-87052011000400015.

Borghezan, M., Gavioli, O., Pit, F. A., \& Silva, A. L. (2010). Modelos matemáticos para a estimativa da área foliar de variedades de videira à campo (Vitis vinifera L.). Ciência Técnica Vitivinicultura, 25, 1-7. Recuperado em 03 de novembro de 2014, de http://www.scielo. gpeari.mctes.pt/pdf/ctv/v25n1/v25n1a01.pdf

Busato, C., Fontes, P. C. R., Braun, H., \& Busato, C. C. M. (2010). Estimativa da área foliar da batateira, cultivar Atlantic, utilizando dimensôes lineares. Revista Ciência Agronômica, 41, 702-708. http:// dx.doi.org/10.1590/S1806-66902010000400026.

Cargnelutti, A., Fo., Toebe, M., Burin, C., Fick, A. L., Neu, I. M. M., \& Facco, G. (2012a). Estimação da área foliar de mucuna cinza por meio de método não destrutivo. Ciência Rural, 42, 238-242. http:// dx.doi.org/10.1590/0103-8478cr20140551. 
Cargnelutti, A., Fo., Toebe, M., Burin, C., Fick, A. L., \& Casarotto, G. (2012b). Estimativa da área foliar de nabo forrageiro em função de dimensôes foliares. Bragantia, 71, 47-51. http://dx.doi.org/10.1590/ S0006-87052012000100008.

Chavarria, G., Tomm, G. O., Muller, A., Mendonça, H. F., Mello, N., \& Betto, M. S. (2011). Índice de área foliar em canola cultivada sob variaçóes de espaçamento e de densidade de semeadura. Ciência Rural, 41, 2084-2089. http://dx.doi.org/10.1590/S0103-84782011001200008.

Flumignan, D. L., Adami, M., \& Faria, R. T. (2008). Área foliar de folhas íntegras e danificadas de cafeeiro determinada por dimensóes foliares e imagem digital. Coffee Science, 3, 1-6. Recuperado em 03 de novembro de 2014, de http://www.coffeescience.ufla.br/index. php/Coffeescience/article/view/67/133

Godoy, L. J. G., Yanagiwara, R. S., Villas Bôas, R. L. V., Backes, C., \& Lima, C. P. (2007). Análise da imagem digital para estimativa da área foliar em plantas de laranja "Pêra". Revista Brasileira de Fruticultura, 29, 420-424. http://dx.doi.org/10.1590/S0100-29452007000300004.

Heldwein, A. B., Buriol, G. A., \& Streck, N. A. (2009). O clima de Santa Maria. Ciência \& Ambiente, 38, 43-58.

Jandel Scientific (1991). SigmaStat: Statistical software for windows: user's manual. California: Jandel Scientific. 280 p.

Lima, C. J. G. S., Oliveira, F. A., Medeiros, J. F., Oliveira, M. K. T., \& Oliveira, A. F., Fo. (2008). Modelos matemáticos para estimativa de área foliar de feijão-caupi. Revista Caatinga, 21, 120-127. Recuperado em 03 de novembro de 2014, de http://periodicos.ufersa.edu.br/ revistas/index.php/sistema/article/viewFile/587/267

Maldaner, I. C., Heldwein, A. B., Loose, L. H., Lucas, D. D. P., Guse, F. I., \& Bortoluzzi, M. P. (2009). Modelos de determinação nãodestrutiva da área foliar em girassol. Ciência Rural, 39, 1356-1361. http://dx.doi.org/10.1590/S0103-84782009000500008.

Marcolini, M. W., Cecílio, A. B., Fo., \& Barbosa, J. C. (2005). Equaçóes de regressão para a estimativa da área foliar de couve-folha. Científica, 33, 192-198. Recuperado em 03 de novembro de 2014, de http://cientifica.org.br/index.php/cientifica/article/view/53

Nascimento, I. B., Farias, C. H. A., Silva, M. C. C., Medeiros, J. F., Espínola, J., So., \& Negreiros, M. Z. (2002). Estimativa da área foliar do meloeiro. Horticultura Brasileira, 20, 555-558. http://dx.doi. org/10.1590/S0102-05362002000400009.

Pinto, A. C. R., Graziano, T. T., Barbosa, J. C., \& Lasmar, F. B. (2008). Modelos para estimativa da área foliar de Curcuma alismatifolia e Curcuma zedoaria. Bragantia, 67, 549-552. http://dx.doi.org/10.1590/ S0006-87052008000200033.

Santos, H. G., Jacomine, P. K. T., Anjos, L. H. C., Oliveira, V. A., Oliveira, J. B., Coelho, M. R., Lumbreras, J. F., \& Cunha, T. J. F. (2006). Sistema brasileiro de classificaçáo de solos (2 ed.). Rio de Janeiro: Embrapa Solos. 306 p.

Severino, L. S., Vale, L. S., \& Beltrão, N. E. M. (2007). Simple method for measurement of Jatropha curcas leaf area. Revista Brasileira de Oleaginosas e Fibrosas, 11, 9-14. Recuperado em 03 de novembro de 2014, de www.cnpa.embrapa.br/ojs/index.php/RBOF/article/ view/54/62

Statsoft (2005). Statistica 7.0 Software. Tucksa, USA: Statsoft

Toebe, M., Brum, B., Lopes, S. J., Cargnelutti, A., Fo., \& Silveira, T. R. (2010). Estimativa da área foliar de Crambe abyssinica por discos foliares e por fotos digitais. Ciência Rural, 40, 475-478. http://dx.doi. org/10.1590/S0103-84782010000200036.

Toebe, M., Cargnelutti, A., Fo., Burin, C., Fick, A. L., Neu, I. M. M., Casarotto, G., \& Alves, B. M. (2012). Modelos para a estimação da área foliar de feijão de porco por dimensões foliares. Bragantia, 71, 37-41. http://dx.doi.org/10.1590/S0006-87052012005000010.

Tomm, G. O., Wiethölter, S., Dalmago, G. A., \& Santos, H. P. (2009). Tecnologia para produção de canola no Rio Grande do Sul (Embrapa Trigo. Documentos Online, 113). Passo Fundo: Embrapa Trigo. 41 p. Recuperado em 03 de novembro de 2014, de http://www.cnpt. embrapa.br/biblio/do/p_do113.htm

Willmott, C. J. (1981). On the validation of models. Physical Geography, 2, 184-194.

Zucoloto, M., Lima, J. S. S., \& Coelho, R. I. (2008). Modelo matemático para estimativa da área foliar total de bananeira 'PrataAnã’. Revista Brasileira de Fruticultura, 30, 1152-1154. http://dx.doi. org/10.1590/S0100-29452008000400050. 